\title{
Analysis of Adaptation Process of Evidence-Based Interventions by Taking Post-Stroke Dysphagia Screening As an Example-- Based on a Series of Empirical Studies
}

\author{
Xiao-Yan ZHANG ${ }^{1,2,3}$, Jun-Qiang ZHAO ${ }^{4}$, Shang-Qian GAO ${ }^{5}$, Li-Jiao YAN ${ }^{1,2,3}$,
} Xue-Jing $\mathrm{LI}^{1,2,3}$, Jing-Ya MA ${ }^{1,2,3}$, Yu-Fang $\mathrm{HAO}^{1,2,3, *}$

${ }^{1}$ School of Nursing, Beijing University of Chinese Medicine, Beijing, China; ${ }^{2}$ Best Practice Spotlight Organization, Beijing University of Chinese Medicine, Beijing, China; ${ }^{3}$ Collaborating Center of Joanna Briggs Institute, Beijing University of Chinese Medicine, Beijing, China $;{ }^{4}$ School of Nursing, University of Ottawa, Ottawa, Canada;

${ }^{5}$ Department of General Surgery, Peking University First Hospital, Beijing 100034, China

\begin{abstract}
Background: Evidence-based interventions (EBIs) are frequently adapted or modified during the implementation process to meet the needs of the target context, public health professionals, or health practitioners. However, little is known about how the EBIs of the same clinical problem at a different time (sustainability) and a different setting (scalability) should be adapted to facilitate implementation. To address this gap, this research aims to analyze the adaptations process of EBIs by taking post-stroke dysphagia screening as an example based on a series of empirical studies.

Methods: We reviewed three best practice implementation projects of post-stroke dysphagia identification and management in mainland China, and conducted a comparative analysis of three projects of dysphagia screening practical decision-making according to core elements of evidence-based nursing (EBN).

Results: We identified the core elements of EBN that influence decision-making in each best practice implementation project. The decision-making of screening time and tool for dysphagia in the three projects varied. Project 1: Post-stroke dysphagia was screened using Water Swallow Test (WST) before the first intake of liquid or food. Project 2: A sustainability study based on project 1, post-stroke dysphagia was screened using Standardized Swallowing Assessment (SSA) before the first intake of liquid or food, within 24 hours of admission. Project 3: A community scalability study based on project 2, post-stroke dysphagia was screened using Water Swallow Test (WST) before the first intake of liquid or food, within 24 hours after referral.

Conclusions: Adaption of EBIs needs to fully consider the best available external evidence from systematic research, clinical expertise, patient preferences, as well as context.
\end{abstract}

Key words: Adaptation; Evidence-based nursing; Stroke; Dysphagia; Screen; Implementation 


\section{Background}

With the development of evidence-based practice, knowledge transformation and implementation science, emphasis on narrowing the gap between research and practice in medicine, social services, and the public health has led to a prominence of the application of evidencebased interventions ${ }^{[1,2]}$. Policymakers, clinical nurses and even patients need to obtain credible and constantly updated evidence in a timely manner to facilitate nursing decisions and improve service quality. When planning and implementing these interventions, most of the evidence cannot be directly applied to the clinic because the original evidence-based interventions (EBIs) may not match the characteristics of the relevant population, executing agency, and community ${ }^{[3]}$. Evidence similar to "raw materials" needs to be transformed and adapted before it can be applied to relevant populations and implementing agencies ${ }^{[4]}$.

The concept of adaptation was originally proposed by Rogers ${ }^{[5]}$, who defined adaptation as the extent to which innovation was modified during the adoption and implementation process. Other definitions have evolved in the era of EBIs and the emergence of adaptation frameworks ${ }^{[6,7]}$. These definitions share similar characteristics, including deletion, addition or modification of evidence ${ }^{[8]}$ to meet the needs of patient characteristics ${ }^{[9,10]}$, organizational culture ${ }^{[11]}$, constraints such as time or resources ${ }^{[12,13]}$, and accommodating practice or setting circumstances/context ${ }^{[12]}$. In the field of implementing science, the understanding of EBIs adaptation is relatively nascent. In the past evidencebased practice, researchers and practitioners often intentionally or unintentionally conducted evidence adjustments. There are also some frameworks and models that guide the approach to adaptation. Escoffery and his colleagues ${ }^{[14]}$ conducted a scoping study and found 13 adaptation frameworks with 11 program adaptation steps: identifying the research question; identifying relevant studies; selecting studies; charting the data; collating, summarizing, and reporting the results; and consulting with experts. Although it is unclear whether the application of one of the identified frameworks is useful and effective, these frameworks enhance the translation of evidence-based practices ${ }^{[15]}$. A systematic review ${ }^{[16]}$ of adaptations of EBIs found that 42 distinct EBIs were found focusing on HIV/AIDS, mental health, drug abuse, and chronic illnesses. Frequent reasons for adaptation included the need for cultural appropriateness, focusing on a new target population, and implementing in a new setting. Common adaptations were content, context, cultural modifications, and delivery.

However, most of the current adaptions projects are horizontal adaption. How the evidence of the same clinical problem at a different time (sustainability) and a different setting (scalability) should be adapted to facilitate implementation is worthy to be considered. While evidence-based nursing practice projects are expanding the application area horizontally, it is more necessary to carry out a vertical reflection on the completed evidence application projects ${ }^{[17]}$. In the past four years, a series of best practice implementation projects of post-stroke dysphagia identification and management were carried out by evidence-based nursing research center of Beijing University of Chinese Medicine since 2015. This study reviewed these best practice implementation projects, analyzed the adaptations process of evidence at a different time (sustainability) and a different setting (scalability) by taking "screening of dysphagia following stroke (time and tool)" as the entry point, In order to provide in-depth thinking for relevant evidence-based adaptation research and nursing decision-making.

\section{Materials and Methods}

We took the three empirical projects of post-stroke dysphagia identification and management carried out by evidence-based nursing research center of Beijing University of Chinese Medicine as the research objects. The three series of studies are introduced as follows.

Project 1 (an evidence-based nursing practice study on the recognition and management of dysphagia following stroke) ${ }^{[18]}$ : To address the gap between research and practice, we used the Iowa model of evidence-based practice as a guide, searched related 
guidelines, recommended high quality guidelines as the basis of evidence-based practice on post-stroke dysphagia recognition and management, formed the initial recommendation items pool, completed the guideline applicability and clinical practice status assessment. According to the audit results and the guideline implementation barriers and promoting factors analysis results of the experimental unit, formulated the corresponding action strategy plan which was operated for 3 months, eventually achieved an excellent effect in patients' quality of life, satisfaction level, length of stay, and knowledge level of nurses.

Project 2 (the continuous quality improvement of an evidence implementation project--post-stroke dysphagia identification and management: an implementation research $)^{[19]}$ : One year after the implementation of project 1 , we found that the overall implementation rate was significantly lower than that of the previous period. In order to promote the continuity of the original research department project, we took the evidence-based continued quality improvement framework as the guiding framework and referred to the National Health Service Sustainability Model, took the project implementation ward as the research object. Based on the combination of the best available evidence, influencing factors on sustainability and expert consensus meeting, we developed post- stroke dysphagia identification and management project sustainability action plan, and successfully promoted the sustainability of the project 1 .

Project 3 (the community scaling up of a best evidence implementation project--post-stroke dysphagia identification and management: an implementation research): Due to the shortage of medical resources, economic burden and other realistic conditions, about $80 \%$ of stroke patients return to the community and family after they were in stable condition in China ${ }^{[20]}$, but most of them still have dysphagia. In order to translate the results to benefit more patients, we conducted a community scaling up study on the best practice implementation project of stroke dysphagia identification and management. We took the scaling up model of World Health Organization (WHO) as the theoretical support ${ }^{[21]}$, investigated the present situation of a community service institution in Beijing, analyzed the barriers and promotion factors, developed appropriate action strategies to expand the scale.

We reviewed these three best practice implementation projects and conducted a comparative analysis of three projects of dysphagia screening time and tool practical decision-making according to core elements of evidencebased nursing. Data were extracted independently by two evaluators. In case of disagreement, a third party will be

Table 1 Basic information of the best practice implementation projects

\begin{tabular}{|c|c|c|c|c|}
\hline $\begin{array}{l}\text { Series } \\
\text { project }\end{array}$ & Time & Title & Setting & Research Content \\
\hline Project $1^{[18]}$ & $\begin{array}{l}2015- \\
2016\end{array}$ & $\begin{array}{l}\text { An Evidence-based Nursing } \\
\text { Practice Study on the Recognition } \\
\text { and Management of Dysphagia in } \\
\text { Stroke }\end{array}$ & $\begin{array}{l}\text { A 3A-level } \\
\text { general } \\
\text { hospital in } \\
\text { Beijing }\end{array}$ & $\begin{array}{l}\text { To construct and evaluate the evidence- } \\
\text { based clinical practice project for } \\
\text { identification and management of } \\
\text { dysphagia } \\
\text { following stroke }\end{array}$ \\
\hline Project $2^{[19]}$ & $\begin{array}{l}2017- \\
2018\end{array}$ & $\begin{array}{l}\text { The Continuous Quality } \\
\text { Improvement of an Evidence } \\
\text { Implementation Project---Post- } \\
\text { stroke Dysphagia Identification } \\
\text { and Management: an } \\
\text { Implementation Research }\end{array}$ & $\begin{array}{l}\text { A 3A-level } \\
\text { general } \\
\text { hospita in } \\
\text { Beijing }\end{array}$ & $\begin{array}{l}\text { To promote the sustainability application } \\
\text { of Project } 1 \text { based on the evidence } \\
\text { update and factors influencing the } \\
\text { sustainability of the Project } 1\end{array}$ \\
\hline Project 3 & $\begin{array}{l}2019- \\
\text { Now }\end{array}$ & $\begin{array}{l}\text { The Community Scaling Up of } \\
\text { a Best Evidence Implementation } \\
\text { Project--Post-Stroke Dysphagia } \\
\text { Identification and Management: } \\
\text { an Implementation Research }\end{array}$ & $\begin{array}{l}\text { A community } \\
\text { in Beijing }\end{array}$ & $\begin{array}{l}\text { To promote the scale-up of the Project } 2 \\
\text { based on the evidence update and factors } \\
\text { influencing the scaling up of the Project } \\
2\end{array}$ \\
\hline
\end{tabular}




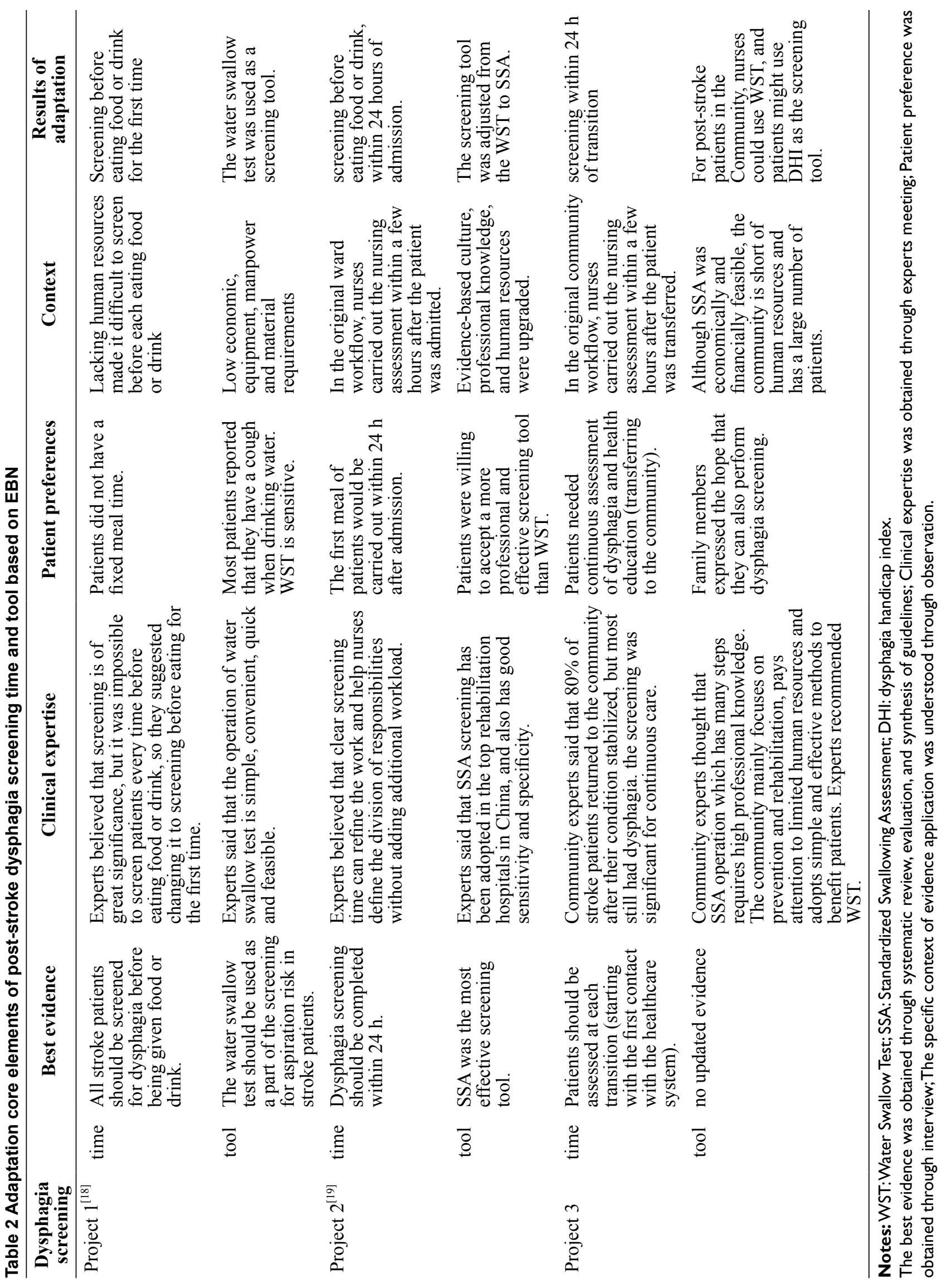


consulted to assist the judgment. After data extraction, we checked the data with the project researcher to make sure the data is correct. Data extraction content mainly included: (1) the basic information of projects, including year, researcher, topic, setting, research content; (2) Stroke dysphagia screening evidence-based practice core elements, including the best evidence, clinical expertise, patient preferences, and context. A completed reporting checklist for updated clinical guidelines, which indicates the sections of the paper where each reporting item is addressed, is available in Table 2.

\section{Results}

We identified the basic information (Table 1) of the best practice implementation projects and the core elements (Table 2) of evidence-based nursing (EBN) that influence decision-making in each best practice implementation project.

\section{The best evidence adaptation process of post- stroke dysphagia screening time}

For the screening time adaptation process, in project 1, the best evidence showed that all stroke patients should be screened for dysphagia before being given food or drink ${ }^{[22]}$. Experts agreed that screening is of great significance, but it was impossible to screen patients every time before eating food or drink with lacking human resources, patients also did not have a fixed meal time, so clinical experts suggested changing this evidence to screening before eating for the first time. In project 2 , as the best evidence was updated, two guidelines ${ }^{[23,24]}$ required patients to be screened within 24 hours of admission and one $^{[25]}$ required screening within an hour of admission. Those guidelines emphasized the importance of early screening. Experts believed that clear screening time can refine the work and help nurses define the division of responsibilities without adding additional workload. The first meal of patients would be done within $24 \mathrm{~h}$ after admission. In the original ward workflow, nurses carried out the nursing assessment within a few hours after the patient was admitted. Therefore, the best evidence had not been substantially adjusted. Evidence adaptation is to screen before eating food or drink within
24 hours of admission. In project 3, when screening for dysphagia was introduced into the new community, the best evidence showed that stroke patients should be assessed at each transition (starting with the first contact with the healthcare system $)^{[26]}$. However, this evidence did not require a specific time. Community experts said that $80 \%$ of stroke patients returned to the community after their condition stabilized, but most of them still had dysphagia. The screening was significant for continuous care. In the original community workflow, nurses carried out the nursing assessment within a few hours after the patient is transferred. Therefore, evidence was adapted as screening within 24 hours of transition.

\section{The best evidence adaptation process of the post-stroke dysphagia screening tool}

For the screening tool adaptation process, in project 1, the best evidence showed that the water swallow test (WST) should be used as a part of the screening for aspiration risk in stroke patients ${ }^{[22]}$. Experts said that the operation of water swallow test is simple, convenient, quick and feasible. WST had Low economic, equipment, manpower and material requirements. Most patients also reported that they had a cough when drinking water. WST which had good sensitivity was used as a screening tool eventually ${ }^{[27]}$. In project 2 , as the best evidence was updated, standardized swallowing assessment (SSA) was the most effective screening tool. Experts said that SSA screening has been adopted in the top rehabilitation hospitals in China, and also has good sensitivity and specificity. Patients were willing to accept a more professional and effective screening tool than WST. Evidence-based culture, professional knowledge, and human resources were upgraded in the primary ward. Therefore, the screening tool was adjusted from the WST to SSA. In project 3, when screening for dysphagia was introduced into the new community, there was no updated evidence. Community experts thought that SSA operation which has many steps requires high professional knowledge. The community mainly focused on prevention and rehabilitation, paid attention to limited human resources, and adopted simple and effective methods to benefit patients. Although SSA was economically and financially feasible ${ }^{[28]}$, the community was short of 
human resources and had a large number of patients. Therefore, experts recommended WST again. In addition, family members expressed the hope that they can also perform dysphagia screening. In conclusion, evidence was adapted as "for post-stroke patients in the community, nurses could use WST, and patients might use dysphagia handicap index (DHI) as the screening tool" ${ }^{[29]}$.

\section{Discussion}

\section{Significance of nurse-led screening for post- stroke dysphagia}

China bears the biggest stroke burden in the world ${ }^{[30]}$. Dysphagia is a common post-stroke symptom and leads to serious complications such as aspiration pneumonia $^{[31]}$. Dysphagia Screening is different from a clinical comprehensive assessment. Compared with the latter, screening is simpler, safer and more economical. Clinical nurses are trained to be competent, and dysphagia screening has high sensitivity and negative predictive value. Relevant evidence also shows that nurse-led screening for dysphagia can reduce lung infection and improve the detection rate of dysphagia (Class A), possibly shortening hospital stay and fasting time (Class $B)^{[32]}$. In the management of patients with dysphagia, The American Heart Association(AHA)/American Stroke Association (ASA) 2016 edition guide ${ }^{[33]}$ recommends the implementation of an oral hygiene management program to reduce the risk of post-stroke aspiration pneumonia (Class I; Level B). The quality and extent of oral hygiene management are also important predictors of aspiration pneumonia. Meanwhile, AHA/ASA also treats behavioral intervention (appropriate dietary adjustment, swallowing rehabilitation, environmental adjustment, safe swallowing) as part of dysphagia treatment (Class IIb; Level A). In addition, nurses play an important role in compensatory methods (posture adjustment and dietary changes), non-oral diet (enteral nutrition), nutrition management, psychological care, patients and caregiver health education ${ }^{[34]}$.

\section{Significance of adaptation to evidence-based nursing practice}

Evidence-based practice emphasizes the promotion of clinical practice based on the best evidence. At present, evidence-based clinical practice guides in the field of nursing at home and abroad are very rich in resources ${ }^{[35-36]}$. Guide refines specific problems and forms recommendations for interventions related to the clinical situation, which has high application value for clinical decision-making. However, there is a huge gap between sufficient evidence and clinical practice, except for resources and capacity constraints, a very important reason is that clinical nurses get relevant recommendations but do not know how to operate, how to make care decisions between the pros and cons, and finally integrate the evidence with clinical practice. In other words, although the evidence is hierarchical, having a high level of evidence does not mean a high recommendation level, and a lower quality level does not necessarily have a low recommendation level ${ }^{[37]}$. How to make clinical decisions based on evidence is also worth exploring. Therefore, evidence adaptation is designed to find the best balance among patients, caregivers, clinical context and evidence, and is of great importance for evidence-based care practices, knowledge transfer, and implementation science.

\section{Adaptation of EBI should be combined with the clinical expertise}

Clinical expertise is the sensitivity of the nursing staff to clinical problems, as well as the application of rich clinical knowledge and experience, professional clinical skills to make specialized decision-making. As a key stakeholder in evidence-based nursing practice, clinical experts are keen to combine evidence with clinical practice issues and give constructive advice. Evidencebased practice is a process of clinical change, which will change the working mode and process of nurses for many years. In practice, multiple obstacles are encountered, and clinical nurses can use their rich experience and practical skills to prejudge the obstacles that evidence will face in the implementation process. For example, in this research project 3 , if the SSA in the second project is directly introduced into the community, regardless of the professional judgment and acceptance of the nursing staff, in the face of the cumbersome and time-consuming screening tools and numerous patients, the community 
nursing staff will eventually become negative, there will also be rejection of change projects. Therefore, evidencebased practice projects must consider the professional judgment, willingness, and acceptance of nurses ${ }^{[38]}$.

\section{Adaptation of EBI should be combined with the patient preferences}

Evidence-based nursing practices emphasize the importance of evidence, but nursing decision makers must be clear that although evidence-based decisions are logically more scientific and effective than decisions without evidence, the evidence itself does not equal or determine care decisions. Patient needs and preferences are at the heart of evidence-based decision making ${ }^{[39]}$. The modern concept of nursing also emphasizes providing compassionate, personalized, and humanistic care services based on respect for patient preferences, values, need and promoting shared decision making (SDM $)^{[40]}$. SDM, a structured form of patient-centered communication, includes information exchange, deliberation, and consensus building and is advocated in clinical situations where the evidence leaves room for more than one medically reasonable choice. SDM bridges patientcentered nursing with evidence-based nursing, while at the same time highlighting the tensions between them. Due to the differences in family background, degree of illness, personal experience, values, medical insurance, and knowledge of the disease, post-stroke patients may refuse to fully demonstrate effective measures because of financial burden or may insist on expensive and ethical and value-based invalid treatment. The process of evidence-based nursing practice is not only the process of treating and nursing diseases but also the process of fully reflecting people's natural attributes, social attributes and meeting the needs of patients. The values of patients should be respected and the patients should be provided with sufficient information.

\section{Adaptation of EBI should be combined with the context}

In our study, the focus of the hospital setting and community setting on the adaptation of evidence for dysphagia screening varies. A new evidence-based practice can be implemented in one environment, but in fact, it fails to be implemented in another environment. The resource distribution, conditions, cultural customs, organizational atmosphere and other aspects of different institutions are different, which will affect the application of evidence from varying degrees. Evidence application projects must consider specific clinical scenarios. The development of the projects should be based on the evaluation of clinical practice, and the suitability of clinical context evidence should also be considered. Explaining from another aspect, the applicability of evidence depends to a large extent on the characteristics of evidence transformed into clinical practice programs and the characteristics of clinical changes ${ }^{[4]}$. It is usually divided into environmental suitability, the safety of evidence application, expected benefits, and feasibility in a variable environment. Only in combination with specific clinical context can we better promote evidence-based practice change. In addition, how to introduce evidencebased practice interventions to new people or new health care systems is also an area of international concern. Relevant scholars have also proposed some concepts, theories, and models in this field, which are worthy of reference for evidence-based practitioners ${ }^{[41-43]}$.

\section{Conclusion}

In summary, this study retrospectively analyzed three consecutive evidence-based practice projects for the identification and management of dysphagia in post-stroke and took the dysphagia screening (time and tools) as the starting point to explore the adaptations process of EBIs based on core elements of EBN. Adaptations of EBI are occurring in natural settings for a variety of reasons, and commonly, adaptations are made to intervention content or context. A few steps were used across adaptation teams in the process of adaptations, but the science of adaptation is still an emerging area of study in implementation science. Adaption of EBIs needs to fully consider the best available external evidence from systematic research, clinical expertise, patient preferences, as well as context. More critical appraisal of intervention adaptations and their outcomes could assist with EBI transferability to increase the scale up and spread of EBI to increase population health impact. 


\section{Acknowledgements}

We thank the participants in the three best practices projects, for their valuable feedback.

\section{Declaration}

The authors of this article declare no conflict of interest.

\section{References}

1 Zhong J, Zhou YF. Progress in methodology and application of implementation research. Chin J Nurs, 2018, 53(7): 862-866.

2 Zhou YF, Hu Y, Gu YH, et al. Application of Knowledge translation models in evidence-based nursing practice. J Nurs Sci, 2016, 31(2): 84-87.

3 Card JJ, Solomon J, Cunningham SD. How to adapt effective programs for use in new contexts. Health Promot Pract, 2011, 12:25-35.

4 Brown SJ. Evidence-based Nursing: the ResearchPractice Connection. Burlington: Jones \& Bartlett Learning, 2012.

5 Rogers E. Diffusion of Innovations. New York: Simon \& Schuster, 1995

6 Lee SJ, Altschul I, Mowbray CT. Using planned adaptation to implement evidence-based programs with new populations. Am J Community Psychol, 2008, 41(3-4): 290-303.

7 Smith E, Caldwell L. Adapting evidence-based programs to new contexts: what needs to be changed? J Rural Health, 2007, 23(Suppl 1): 37-41.

8 Storer HL, Barkan SE, Sherman EL, et al. Promoting Relationship Building and Connection: Adapting an Evidence-Based Parenting Program for Families Involved in the Child Welfare System. Child Youth Serv Rev, 2012, 34(9): 1853-1861.

9 Carvalho ML, Honeycutt S, Escoffery C, et al. Balancing fidelity and adaptation: implementing evidence-based chronic disease prevention programs. J Public Health Manag Pract, 2013, 19(4): 348-56.

10 Cooper BR, Shrestha G, Hyman L, et al. Adaptations in a community-based family intervention: replication of two coding schemes. J Prim Prev, 2016, 37(1): 33-
52.

11 Moore JE, Bumbarger BK, Cooper BR. Examining adaptations of evidence-based programs in natural contexts. J Prim Prev, 2013, 34(3): 147-61.

12 Cohen DJ, Crabtree BF, Etz RS, et al. Fidelity versus flexibility: translating evidence-based research into practice. Am J Prev Med, 2008, 35(Suppl 5): S381-S389.

13 Miller-Day M, Pettigrew J, Hecht ML, et al. How prevention curricula are taught under realworld conditions: types of and reasons for teacher curriculum adaptations. Health Educ, 2013, 113(4): 324-344.

14 Escoffery C, Lebow-Skelley E, Udelson H, et al. A scoping study of frameworks for adapting public health evidence-based interventions. Transl Behav Med, 2019, 9(1): 1-10.

15 Wandersman A, Duffy J, Flaspohler P, et al. Bridging the gap between prevention research and practice: the interactive systems framework for dissemination and implementation. Am J Community Psychol, 2008, 41(3-4): 171-181.

16 Escoffery C, Lebow-Skelley E, Haardoerfer R, et al. A systematic review of adaptations of evidence-based public health interventions globally. Implement Sci, 2018, 13(1): 125 .

17 Cheng L, Feng S, Hu Y. Evidence-based nursing implementation in Mainland China: A scoping review. Nurs Outlook, 2016, 65(1): 27-35.

18 Gao SQ. An Evidence-based Nursing Practice Study on the Recognition and Management of Dysphagia in Stroke. Beijing: Beijing University of Chinese Medicine, 2016.

19 Zhao JQ. The Continuous Quality Improvement of an Evidence Implementation Project---Post-stroke Dysphagia Identification and Management: an Implementation Research. Beijing: Beijing University of Chinese Medicine, 2018.

20 Shi $\mathrm{D}, \mathrm{Li} \mathrm{Z}$. Interventions to reduce caregiving burden of family caregivers of stroke patients: a literature review. J Nurs Sci, 2016, 31(17): 107-110.

21 World Health Organization. Nine steps for developing a scaling-up strategy. [article online], 2010. Available from: http://www.expandnet.net/PDFs/ExpandNet- 
WHO\%20Nine\%20Step\%20Guide\%20published.pdf. [Accessed on October 22, 2018].

22 Scottish Intercollegiate Guidelines Network (SIGN). Management of patients with stroke: Identification and management of dysphagia. Edinburgh: SIGN, 2010.

23 Registered Nurses Association of Ontario (RNAO). Stroke assessment across the continuum of care. [article online], 2011. Available from: https:// bpgmobile.rnao.ca/guideline-content/24. [Accessed on November, 2018].

24 Winstein CJ, Stein J, Arena R, et al. Guidelines for adult stroke rehabilitation and recovery: a guideline for healthcare professionals from the American Heart Association/American Stroke Association. Stroke, 2016, 47(6): e98-e100.

25 Wirth R, Smoliner C, Jager M, et al. Guideline clinical nutrition in patients with stroke. Exp Transl Stroke Med, 2013, 5(1): 14-16.

26 Cameron JI, O'Connell C, Foley N, et al. Canadian Stroke Best Practice Recommendations: Managing transitions of care following Stroke, Guidelines Update 2016. Int J Stroke, 2016, 11(7): 807-822.

$27 \mathrm{Wu}$ SL, Ma C, Huang FY, et al. Clinical application of standardized swallowing assessment. Chin J Phys Med Rehabil, 2008, 30(6): 396-399.

28 Dou ZL. Evaluation and treatment of dysphagia. Beijing: People's Health Publishing House, 2017 : 123-124.

29 China Association of Geriatrics Nutrition and Food Safety Branch. China expert consensus on home nutrition administration for elderly patients with dysphagia. Chin J Evid-based Med, 2018, 18(6): 547558.

30 Wang W, Jiang B, Sun H, et al. Prevalence, Incidence, and Mortality of Stroke in China. Circulation, 2017, 135(8): 759.

31 Palli C, Fandler S, Doppelhofer K, et al. Early Dysphagia Screening by Trained Nurses Reduces Pneumonia Rate in Stroke Patients: A Clinical Intervention Study. Stroke, 2017, 48(9): 2583-2585.
32 Hines S, Kynoch K, Munday J. Identification and nursing management of dysphagia in individual with acute neurological impairment: a systematic review (new update). JBI Database System Rev Implement Rep, 2014, 12(5): 195-236.

33 Zhang XY, Jiang H. 2016 Edition of Adult Stroke Rehabilitation Guide Interpretation: Nursing for Dysphagia. Shanghai Nurs, 2018, 18(8): 5-8.

34 Travers PL. Poststroke Dysphagia: Implications for Nurses. Rehabil Nurs, 2012, 24(2): 69-73.

$35 \mathrm{Hu} \mathrm{Y,} \mathrm{Zhu} \mathrm{Z,} \mathrm{Fu} \mathrm{L,} \mathrm{Cheng} \mathrm{L,} \mathrm{et} \mathrm{al.} \mathrm{Evidence-based}$ nursing based on clinical practice guideline practice and reflection. Chin Nurs Manag, 2016, 16(9): 11561160.

36 Liu J, Liu DD, Shi Y, et al. Quality evaluation of clinical practice guidelines for nursing at home and abroad from 2013 to 2018. J Nurses Training, 2019, 34(6):488-497.

37 Cheng YL, Li YP, Du L, et al. Evolution of Levels of Evidence and Strength of Recommendations in Medical Research. Chin J Evid-Based Med, 2008, 8(2): 127-133.

38 Yuan HT, Li L, Zeng TY. Research advances in barriers to evidence-based nursing practice and countermeasures. J Nurs Sci, 2013, 28(21): 94-97.

$39 \mathrm{Hu} \mathrm{Y,} \mathrm{Hao} \mathrm{YF.} \mathrm{Evidence-Based} \mathrm{Nursing.} \mathrm{Beijing:}$ People's Medical Publishing House, 2018.

40 Adams JR, Drake RE. Shared Decision-Making and Evidence-Based Practice. Community Ment Health J, 2006, 42(1): 87-105.

41 Aarons GA, Sklar M, Mustanski B, et al. "Scalingout" evidence-based interventions to new populations or new health care delivery systems. Implement Sci, 2017, 12(1): 111.

42 Ben CA, Zomahoun HTV, LeBlanc A, et al. Effective strategies for scaling up evidence-based practices in primary care: a systematic review. Implement Sci, 2017, 12(1): 139.

43 Milat AJ, Bauman A, Redman S. Narrative review of models and success factors for scaling up public health interventions. Implement Sci, 2015, 10(1): 113. 